\title{
A Nomogram for Prediction of Postoperative Pneumonia Risk in Elderly Hip Fracture Patients
}

This article was published in the following Dove Press journal:

Risk Management and Healthcare Policy

\author{
Guangheng Xiang ${ }^{1,2, *}$ \\ Xiaoyu Dong (iD ${ }^{1, *}$ \\ Tao Xu' \\ Yongzeng Feng' \\ Zili $\mathrm{He}^{\prime}$ \\ Chenrong $\mathrm{Ke}^{\mathrm{I}}$ \\ Jian Xiao ${ }^{1,2}$ \\ Yi-Min Weng'
}

'Department of Orthopaedic, The Second Affiliated Hospital and Yuying Children's Hospital of Wenzhou Medical University, Wenzhou, Zhejiang 325035,

People's Republic of China; ${ }^{2}$ School of Pharmaceutical Sciences, Wenzhou

Medical University, Wenzhou, Zhejiang

325035, People's Republic of China

*These authors contributed equally to this work
Correspondence: Jian Xiao; Yi-Min Weng Department of Orthopaedic, The Second Affiliated Hospital and Yuying Children's Hospital of Wenzhou Medical University, Wenzhou, Zhejiang 325035, People's

Republic of China

Email xfxj2000@I26.com;

wzykdxwym@163.com
Purpose: Pneumonia is one of the common complications of hip fracture. This study aimed to evaluate the risk factors and apply a nomogram to predict postoperative pneumonia in elderly hip fracture patients.

Materials and Methods: From August 2014 to October 2019, 1113 hip fracture patients who were older than 65 years and underwent surgical treatment in our hospital were subjects of this study. Univariate and multivariate Cox analyses were used to identify independent risk factors. A predictive nomogram model was built, and the discrimination and calibration were determined by receiver operating characteristic and calibration plot.

Results: A total of 166 patients developed pneumonia after operation (14.91\%, pneumonia group) while the remaining 947 patients did not (85.09\%, non-pneumonia group). According to the results, body mass index (OR, $0.76,95 \% \mathrm{CI}, 0.70$ to $0.84, \mathrm{P}<0.001$ ), serum albumin (OR, 0.86, 95\% CI, 0.79 to $0.93, \mathrm{P}<0.001$ ), c-reactive protein (OR, 1.01, 95\% CI, 1.00 to 1.92, $\mathrm{P}=0.011)$, functional status $(\mathrm{OR}, 2.94,95 \% \mathrm{CI}, 1.69$ to $5.10, \mathrm{P}<0.001)$ and time to surgery (OR, 4.56, 95\% CI, 2.64 to $7.88, \mathrm{P}<0.001$ ) were identified as independent risk factors of pneumonia. The area under the curve value for postoperative pneumonia risk was 0.905 , and the P-value of the Hosmer-Lemeshow calibration test was 0.529.

Conclusion: Our nomogram model can be used to predict the risk of pneumonia in elderly hip fractures after surgery and provide clinicians with guidance for better perioperative intervention to improve prognosis and reduce mortality.

Keywords: pneumonia, elderly, hip fracture, risk factors, nomogram

\section{Introduction}

At present, osteoporotic hip fractures have become a major health problem, and its devastating consequences included functional decline, institutionalization, and even mortality. ${ }^{1}$ The incidence of osteoporotic hip fractures increases as the population ages, and is predicted to increase by 1.4 times by $2025 .^{2}$ Most elderly patients with hip fractures are treated by surgery because of worsened outcomes without surgical operation.

As many as $30 \%$ of patients die within one year after hip fractures, and onethird of them die in hospital. ${ }^{3}$ The cause of mortality in elderly people with hip fractures is mainly due to postoperative complications such as wound infection, pneumonia, deep vein thrombosis, urinary tract infections, and cardiovascular and cerebrovascular events. ${ }^{4-8}$ Therefore, perioperative management and prevention of postoperative complications have recently become the focus of treatment for geriatric hip fractures. 
Pneumonia is one of the common complications of hip fracture and the incidence is between $5 \%$ and $10 \%{ }^{9-15}$ Most postoperative pneumonia cases are caused by gramnegative aerobic bacteria including Pseudomonas, Klebsiella and Enterobacteriaceae. Given the high incidence of pneumonia in elderly hip fracture patients, it has become increasingly important to identify risk factors of pneumonia. Therefore, early detection of hip fracture patients with a tendency towards developing into pneumonia and implementing the effective intervention therapy can significantly reduce the complications and improve patient prognosis.

The purpose of this study is to determine the risk factors associated with pneumonia after hip fracture surgery and to establish a nomogram model to predict the incidence.

\section{Materials and Methods Inclusion and Exclusion Criteria}

This study followed the guidelines of the "Declaration of Helsinki" and was approved by the hospital's ethics committee (Research Ethics Committee of the Second Affiliated Hospital of Wenzhou Medical University, L-2020-22). The data were analyzed anonymously and personal identifiers were completely removed; therefore, the requirement for informed consent was waived.

We retrospectively collected data from our hospital on geriatric hip fracture patients between August 2014 and October 2019. The eligibility criteria were: 1) femoral intertrochanteric fractures (International Classification of Diseases, 10th Revision [ICD-10] S72.1); 2) caused by a low-energy injury (eg, falls from standing height and osteoporosis); 3 ) ages $\geq 65$ years; 4) recipients of surgeries including open or closed reduction internal fixation. Exclusion criteria included: 1) pathological fractures; 2) multiple fractures or multiple trauma; 3) conservative treatment; 4) loss of data; 5) history of hip fracture; 6) pneumonia occurred before surgery. In the end, there were 1113 patients included in the final analysis (Supplementary Figure).

\section{Diagnosis of Pneumonia}

In brief, the diagnostic criteria for pneumonia referred to "radiologic criteria" (chest radiographs and computed tomography) and "signs/symptoms/laboratory criteria". Postoperative Pneumonia was defined as those who did not have pneumonia before surgery but had new or increased lung infiltration on the chest X-ray and/or computed tomography images after surgery.

\section{Analysis of Risk Factors}

Blood tests were taken on the first day after admission. In our study, patients were characterized at baseline in terms of age, gender, body mass index (BMI), hemoglobin, serum albumin, c-reactive protein (CRP), creatinine, functional status, current smoking status, current drinking status, length of hospital stay, intensive care unit (ICU) admission and in-hospital mortality. The patient's previous medical history was evaluated, including diabetes, hypertension, myocardial infarction, heart failure, arrhythmia, chronic obstructive pulmonary disease (COPD), asthma and malignancy. Factors related to surgery were also assessed, including time to surgery, operative time, blood transfusion, anesthesia type, American Society of Anesthesiologists (ASA) classification.

\section{Statistical Analysis}

Continuous variables were expressed as the means \pm standard deviations, and categorical variables were expressed as percentages. Comparisons between groups were performed using the $\chi^{2}$-test, Fisher's exact test, and Student's $t$-test. Univariate analyses were used to evaluate the associations between different factors and the postoperative pneumonia. In order to determine the independent risk factors, variables achieving a significance of $\mathrm{P}<$ 0.1 were selected for multivariable analyses. Then, based on the regression coefficients of independent variables, we established the nomogram prediction model of postoperative pneumonia. The distinction of dichotomous results was usually evaluated by calculating the area under the curve (AUC) of the receiver operating characteristic (ROC) curve. Generally, a prediction model with an AUC of 0.5-0.75 was considered acceptable, and AUC> 0.75 means that the model shows excellent discriminative. In addition, the decision curve analysis was carried out to assess the net benefit of the nomogram to the decision. Statistical analyses were carried out using $\mathrm{R}$ version 3.6.1 for Windows (R Foundation for Statistical Computing, Vienna, Austria) and EmpowerStats (http://www.empower stats.com, X\&Y Solutions, Inc., Boston, MA). P values less than 0.05 (two-sided) were considered statistically significant. 
Table I Baseline Characteristics

\begin{tabular}{|c|c|c|c|c|}
\hline Variable & Total $(n=|I| 3)$ & Without Pneumonia (n=947) & With Pneumonia $(n=166)$ & P-value* \\
\hline Age, years & $79.9 \pm 7.5$ & $78.8 \pm 7.2$ & $86.4 \pm 5.8$ & $<0.001$ \\
\hline $\mathrm{BMI}, \mathrm{Kg} / \mathrm{m}^{2}$ & $23.6 \pm 3.0$ & $24.1 \pm 2.8$ & $20.6 \pm 2.4$ & $<0.001$ \\
\hline Hemoglobin, g/L & $105.8 \pm 13.8$ & $105.9 \pm 14.0$ & $104.8 \pm 12.8$ & 0.335 \\
\hline Serum albumin, $g / d L$ & $36.7 \pm 3.1$ & $37.3 \pm 2.6$ & $33.3 \pm 3.4$ & $<0.001$ \\
\hline C-reactive protein, $\mathrm{mg} / \mathrm{L}$ & $58.8 \pm 32.3$ & $54.0 \pm 29.2$ & $85.8 \pm 35.5$ & $<0.001$ \\
\hline Creatinine, $\mu \mathrm{mol} / \mathrm{L}$ & $83.6 \pm 67.9$ & $83.6 \pm 64.6$ & $83.9 \pm 84.3$ & 0.950 \\
\hline Operative time, $\min$ & $52.1 \pm 11.4$ & $51.9 \pm 10.8$ & $53.0 \pm 14.1$ & 0.225 \\
\hline Length of hospital stay, days & $9.1 \pm 3.6$ & $8.4 \pm 1.4$ & $13.3 \pm 7.4$ & $<0.001$ \\
\hline Gender & & & & 0.450 \\
\hline Male & $384(34.5 \%)$ & $331(35.0 \%)$ & $53(31.9 \%)$ & \\
\hline Female & 729 (65.5\%) & $616(65.0 \%)$ & $113(68.1 \%)$ & \\
\hline Anesthesia type & & & & 0.240 \\
\hline Regional & $819(73.6 \%)$ & $703(74.2 \%)$ & $116(69.9 \%)$ & \\
\hline General & 294 (26.4\%) & 244 (25.8\%) & $50(30.1 \%)$ & \\
\hline ASA classification & & & & $<0.001$ \\
\hline I & 120 (10.8\%) & 117 (I2.4\%) & $3(1.8 \%)$ & \\
\hline 2 & 549 (49.3\%) & $523(55.2 \%)$ & $26(15.7 \%)$ & \\
\hline 3 & 390 (35.0\%) & $280(29.6 \%)$ & 110 (66.3\%) & \\
\hline 4 & 54 (4.9\%) & $27(2.9 \%)$ & 27 (16.3\%) & \\
\hline Functional status & & & & $<0.001$ \\
\hline Independent & 905 (81.3\%) & $828(87.4 \%)$ & 77 (46.4\%) & \\
\hline Dependent & 208 (18.7\%) & $119(12.6 \%)$ & $89(53.6 \%)$ & \\
\hline Time to surgery & & & & $<0.001$ \\
\hline Less than $48 \mathrm{~h}$ & 635 (57.1\%) & $611(64.5 \%)$ & 24 (14.5\%) & \\
\hline More than $48 \mathrm{~h}$ & 478 (42.9\%) & $336(35.5 \%)$ & 142 (85.5\%) & \\
\hline Smoking & & & & 0.287 \\
\hline No & 911 (81.9\%) & 780 (82.4\%) & $|3|$ (78.9\%) & \\
\hline Yes & 202 (18.1\%) & $167(17.6 \%)$ & 35 (2I.1\%) & \\
\hline Drinking & & & & 0.977 \\
\hline No & 958 (86.1\%) & $815(86.1 \%)$ & $143(86.1 \%)$ & \\
\hline Yes & 155 (13.9\%) & $132(13.9 \%)$ & 23 (13.9\%) & \\
\hline Blood transfusion & & & & 0.970 \\
\hline No & 826 (74.2\%) & $703(74.2 \%)$ & $123(74.1 \%)$ & \\
\hline Yes & 287 (25.8\%) & 244 (25.8\%) & 43 (25.9\%) & \\
\hline Diabetes & & & & 0.512 \\
\hline No & 886 (79.6\%) & 757 (79.9\%) & 129 (77.7\%) & \\
\hline Yes & 227 (20.4\%) & $190(20.1 \%)$ & 37 (22.3\%) & \\
\hline Hypertension & & & & 0.607 \\
\hline No & $536(48.2 \%)$ & 453 (47.8\%) & $83(50.0 \%)$ & \\
\hline Yes & 577 (51.8\%) & 494 (52.2\%) & $83(50.0 \%)$ & \\
\hline
\end{tabular}


Table I (Continued).

\begin{tabular}{|c|c|c|c|c|}
\hline Variable & Total $(n=1113)$ & Without Pneumonia $(n=947)$ & With Pneumonia $(n=166)$ & P-value* \\
\hline Myocardial infarction & & & & 0.518 \\
\hline No & 1062 (95.4\%) & 902 (95.2\%) & 160 (96.4\%) & \\
\hline Yes & $5 I(4.6 \%)$ & 45 (4.8\%) & $6(3.6 \%)$ & \\
\hline Heart failure & & & & 0.721 \\
\hline No & $1060(95.2 \%)$ & 901 (95.1\%) & 159 (95.8\%) & \\
\hline Yes & $53(4.8 \%)$ & $46(4.9 \%)$ & $7(4.2 \%)$ & \\
\hline Arrhythmia & & & & 0.913 \\
\hline No & 1048 (94.2\%) & 892 (94.2\%) & 156 (94.0\%) & \\
\hline Yes & $65(5.8 \%)$ & $55(5.8 \%)$ & $10(6.0 \%)$ & \\
\hline COPD & & & & 0.002 \\
\hline No & 999 (89.8\%) & 861 (90.9\%) & $138(83.1 \%)$ & \\
\hline Yes & $114(10.2 \%)$ & $86(9.1 \%)$ & $28(16.9 \%)$ & \\
\hline Asthma & & & & 0.520 \\
\hline No & IIOI (98.9\%) & $936(98.8 \%)$ & 165 (99.4\%) & \\
\hline Yes & $12(1.1 \%)$ & $936(98.8 \%)$ & 165 (99.4\%) & \\
\hline Malignancy & & & & 0.488 \\
\hline No & $1070(96.1 \%)$ & $912(96.3 \%)$ & $158(95.2 \%)$ & \\
\hline Yes & 43 (3.9\%) & $35(3.7 \%)$ & $8(4.8 \%)$ & \\
\hline ICU admission & & & & $<0.001$ \\
\hline No & 1025 (92.1\%) & 897 (94.7\%) & $128(77.1 \%)$ & \\
\hline Yes & $88(7.9 \%)$ & $50(5.3 \%)$ & 38 (22.9\%) & \\
\hline In-hospital mortality & & & & $<0.001$ \\
\hline No & 1080 (97.0\%) & 931 (98.3\%) & 149 (89.8\%) & \\
\hline Yes & $33(3.0 \%)$ & $16(1.7 \%)$ & $17(10.2 \%)$ & \\
\hline
\end{tabular}

Notes: Data are presented as the mean and the standard deviation with the range in parenthesis or expressed as the number with the percentage in parenthesis. *P-value, differences between patients with pneumonia and control.

Abbreviations: ASA score, American Society of Anesthesiologists score; BMI, body mass index; COPD, chronic obstructive pulmonary disease; ICU, intensive care unit.

\section{Results}

From August 2014 to October 2019, 1113 hip fracture patients who were older than 65 years and underwent surgical treatment in our hospital were subjects of this study. Among them, 166 patients developed pneumonia after operation (14.91\%, pneumonia group) while the remaining 947 patients did not $(85.09 \%$, non-pneumonia group) (Table 1). The average age of patients with pneumonia was $86.4 \pm 5.8$ years, which was significantly higher than the age of the control group $(\mathrm{P}<0.001)$. The ratio of males among patients with pneumonia was $31.9 \%$ (53/166), which was similar to non-pneumonia group $(\mathrm{P}=$ 0.450 ). There was a statistically significant difference in BMI between the pneumonia group and the control group, $20.6 \pm 2.4 \mathrm{Kg} / \mathrm{m}^{2}$ and $24.1 \pm 2.8 \mathrm{Kg} / \mathrm{m}^{2}$, respectively $(\mathrm{P}<$ 0.001). Among patients with pneumonia, the serum albu$\min$ and CRP were $33.3 \pm 3.4 \mathrm{~g} / \mathrm{dL}$ and $85.8 \pm 35.5 \mathrm{mg} / \mathrm{L}$, whereas among the control participants, the serum albumin and CRP were $37.3 \pm 2.6 \mathrm{~g} / \mathrm{dL}$ and $54.0 \pm 29.2 \mathrm{mg} / \mathrm{L}(\mathrm{P}<$ 0.001 and $\mathrm{P}<0.001$, respectively). The ASA classification $1,2,3$, and 4 were $1.8 \%, 15.7 \%, 66.3 \%$ and $16.3 \%$ in pneumonia patients, and $12.4 \%, 55.2 \%, 29.6 \%$ and $2.9 \%$ in non-pneumonia patients $(\mathrm{P}<0.001)$. Compared to pneumonia group, the percentage of patients with the time interval between injury and surgery was $\geq 48 \mathrm{~h}$ was significantly lower in the non-pneumonia group $(84.2 \%$ VS $46.2 \%, \mathrm{P}<0.001)$. The proportion of patients with dependent functional status was significantly higher in the pneumonia patients than in the control group $(53.6 \%$ vs $12.6 \%, \mathrm{P}<0.001)$. In terms of prognostic implications, the elderly patients who developed pneumonia had a longer hospital stay, a higher frequency of ICU admission and a higher mortality rate during hospitalization than patients without pneumonia $(\mathrm{P}<0.001$, Table 1). Other baseline 
Table 2 Multivariable Logistic Regression of Predictors for Postoperative Pneumonia

\begin{tabular}{|c|c|c|c|}
\hline Variable & OR & $95 \% \mathrm{Cl}$ & $P$ value \\
\hline Age, years & 1.03 & $0.99,1.07$ & 0.126 \\
\hline $\mathrm{BMI}, \mathrm{Kg} / \mathrm{m}^{2}$ & 0.76 & $0.70,0.84$ & $<0.001$ \\
\hline Serum albumin, $g / d L$ & 0.86 & $0.79,0.93$ & $<0.001$ \\
\hline C-reactive protein, $\mathrm{mg} / \mathrm{L}$ & 1.01 & $1.00,1.02$ & 0.011 \\
\hline \multicolumn{4}{|l|}{ ASA classification } \\
\hline 1 & Ref. & & \\
\hline 2 & 0.59 & $0.16,2.16$ & 0.423 \\
\hline 3 & 1.46 & $0.39,5.44$ & 0.575 \\
\hline 4 & 0.94 & $0.20,4.53$ & 0.941 \\
\hline \multicolumn{4}{|l|}{ Functional status } \\
\hline Independent & Ref. & & \\
\hline Dependent & 2.94 & $1.69,5.10$ & $<0.001$ \\
\hline \multicolumn{4}{|l|}{ Time to surgery } \\
\hline Less than $48 \mathrm{~h}$ & Ref. & & \\
\hline More than $48 \mathrm{~h}$ & 4.56 & $2.64,7.88$ & $<0.001$ \\
\hline \multicolumn{4}{|l|}{ COPD } \\
\hline No & Ref. & & \\
\hline Yes & 0.76 & $0.37,1.58$ & 0.469 \\
\hline
\end{tabular}

Note: Data are presented as the odds ratio with the confidence interval in parenthesis.

Abbreviations: BMI, body mass index; COPD, chronic obstructive pulmonary disease; OR, odds ratio; Cl, confidence interval.

data between the pneumonia group and non-pneumonia group showed no significant differences.

The results of univariate analysis showed that the significant risk factors were age, BMI, serum albumin, CRP, ASA classification, functional status, time to surgery and COPD ( $\mathrm{P}<0.01)$. After multivariate logistic regression analysis, BMI, serum albumin, CRP, functional status and time to surgery were identified as independent risk factors of pneumonia associated with elderly patients after a hip fracture (Table 2).

Then, we established a nomogram for predicting postoperative pneumonia including the five independent risk factors based on the multivariate logistic regression analysis (Figure 1). To use the nomogram, the points corresponding to each prediction variable were obtained, and then the sum of the points was calculated as the total score, and the predicted risk corresponding to the total score was the probability of postoperative pneumonia. We drew the ROC curve of predicted probability and calculated the
AUC value. The AUC value for postoperative pneumonia risk was 0.905 , suggesting that the nomogram prediction model has an excellent discrimination (Figure 2). The Hosmer-Lemeshow test also showed that the nomogram prediction model had good calibration ability $(\mathrm{P}=0.529$, Figure $3 \mathrm{~A}$ ). We assumed that the predicted probability above the defined threshold would determine the treatment decision for the patients, while the predicted probability below the threshold would generate other decisions. Therefore, we established a decision analysis curve to assess the net benefit of the nomogram to the decision. The result indicated that the nomogram was applicable when the threshold was in the range of 0.03 to 0.76 due to the net benefit (Figure 3B).

\section{Discussion}

Hip fracture is one of the common causes of hospitalization in the elderly patients. Postoperative pneumonia is the main complication of hip fracture surgery in the elderly, 


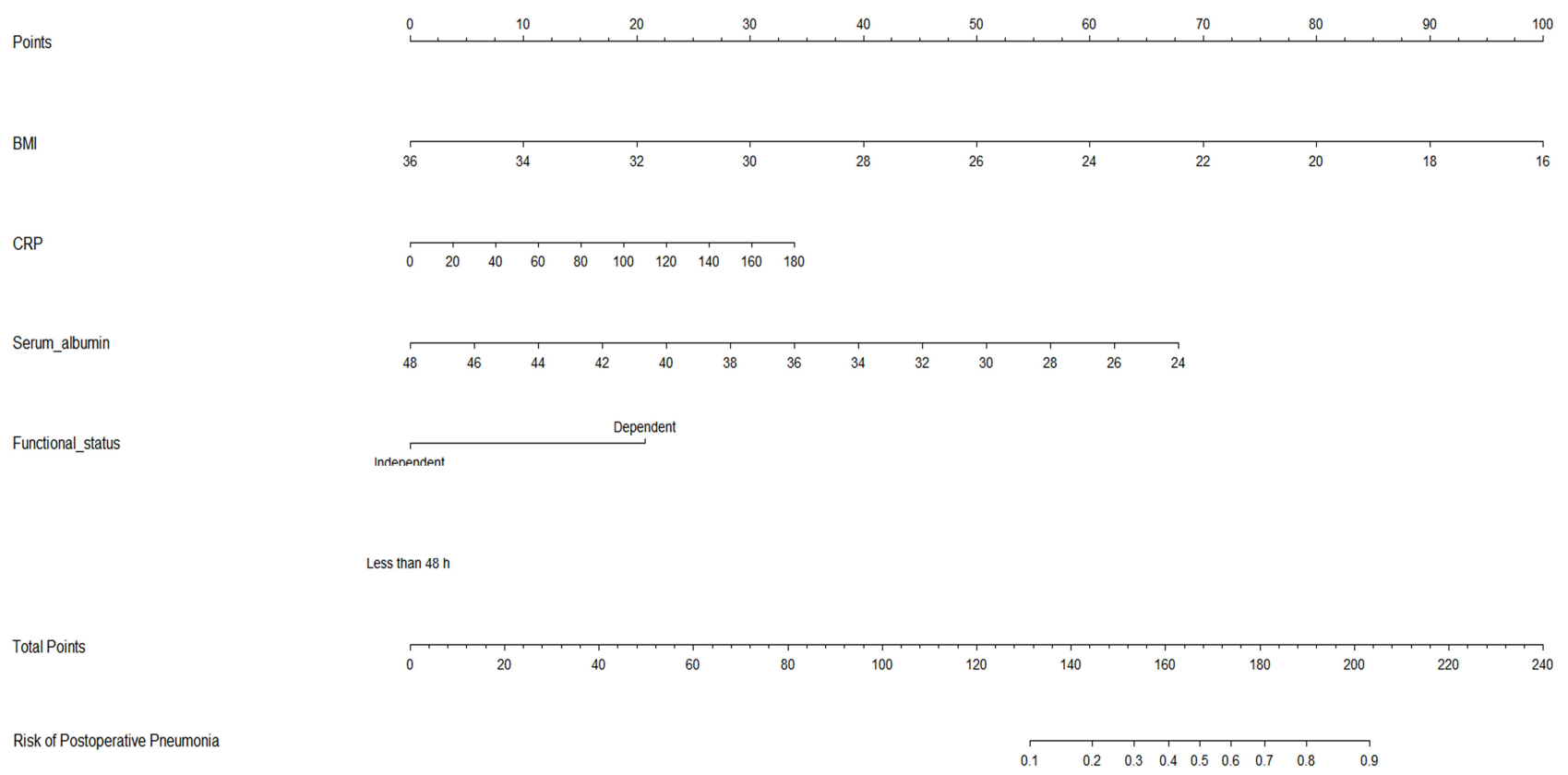

Figure I Predictive nomogram for postoperative pneumonia. To use the nomogram, the points corresponding to each prediction variable were obtained, then the sum of the points was calculated as the total score, and the predicted risk corresponding to the total score was the probability of postoperative pneumonia.

with a mortality rate as high as $30 \%$ to $44 \% .{ }^{16}$ Roche et al showed that postoperative pneumonia was diagnosed in $8.8 \%$ of elderly hip fracture patients, which was the most common complications and resulted in increased mortality. ${ }^{17}$ In a study about geriatric hip fracture complications in 153,613 Canadian patients, the incidence of

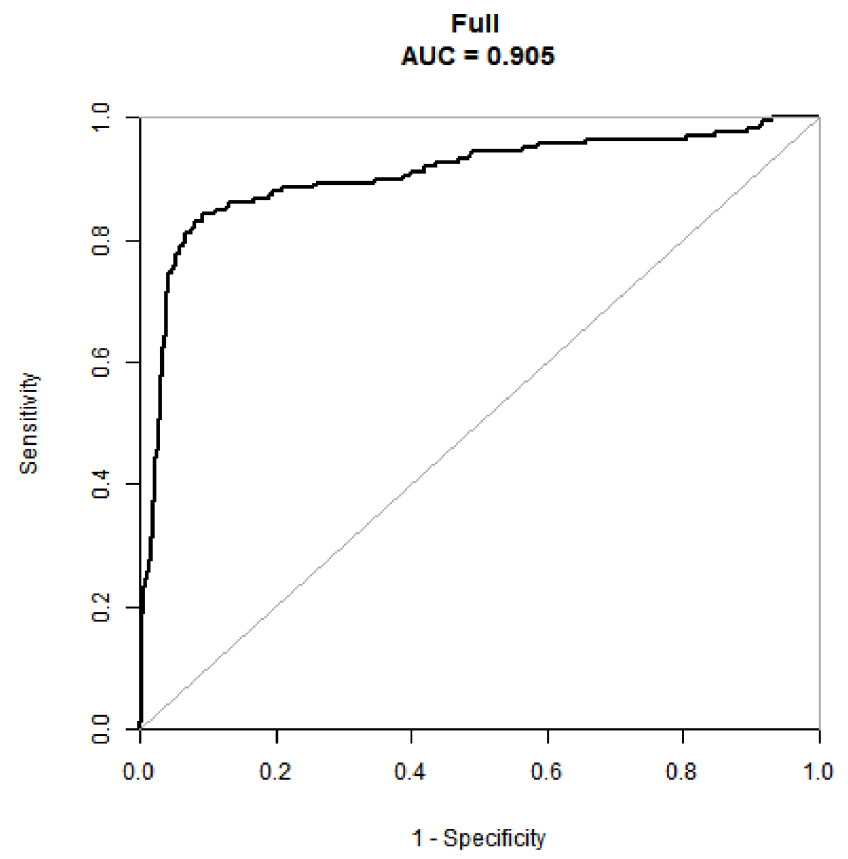

Figure 2 ROC curves for validating the discrimination of the nomogram prediction model. postoperative pneumonia was also $4.9 \%{ }^{18} \mathrm{Lv}$ et al also reviewed 1429 patients who underwent hip fracture surgery in China, and $4.9 \%$ of them developed postoperative pneumonia. ${ }^{13}$ Recently, Jang et al retrospectively analyzed 14,736 Korean hip fracture patients $\geq 65$ years old, $11.05 \%$ of patients suffered from pneumonia during hospitalization. ${ }^{19}$ However, $14.9 \%$ of elderly hip fracture patients were identified as pneumonia after surgery in this study, which was slightly higher than the previous articles. This might be related to the increasing social aging in recent years.

Among elderly hip fracture patients, the risk factors for postoperative pneumonia development remained controversial. Potential risk factors for postoperative pneumonia might include age, gender, BMI, hemoglobin, serum albumin, CRP, functional status, current smoking status, heart failure, COPD, time to surgery, operative time, blood transfusion and ASA classification. ${ }^{12,13,20-23}$ In the present study, BMI, CRP, serum albumin, functional status and time to surgery were identified as independent risk factors for postoperative pneumonia (Table 2). On the other hand, no significant differences were observed in the hemoglobin, creatinine, gender, anesthesia type, blood transfusion, current smoking status, current drinking status, operative time, history of hypertension, history of diabetes, history of circulatory disease and history of malignancy. 

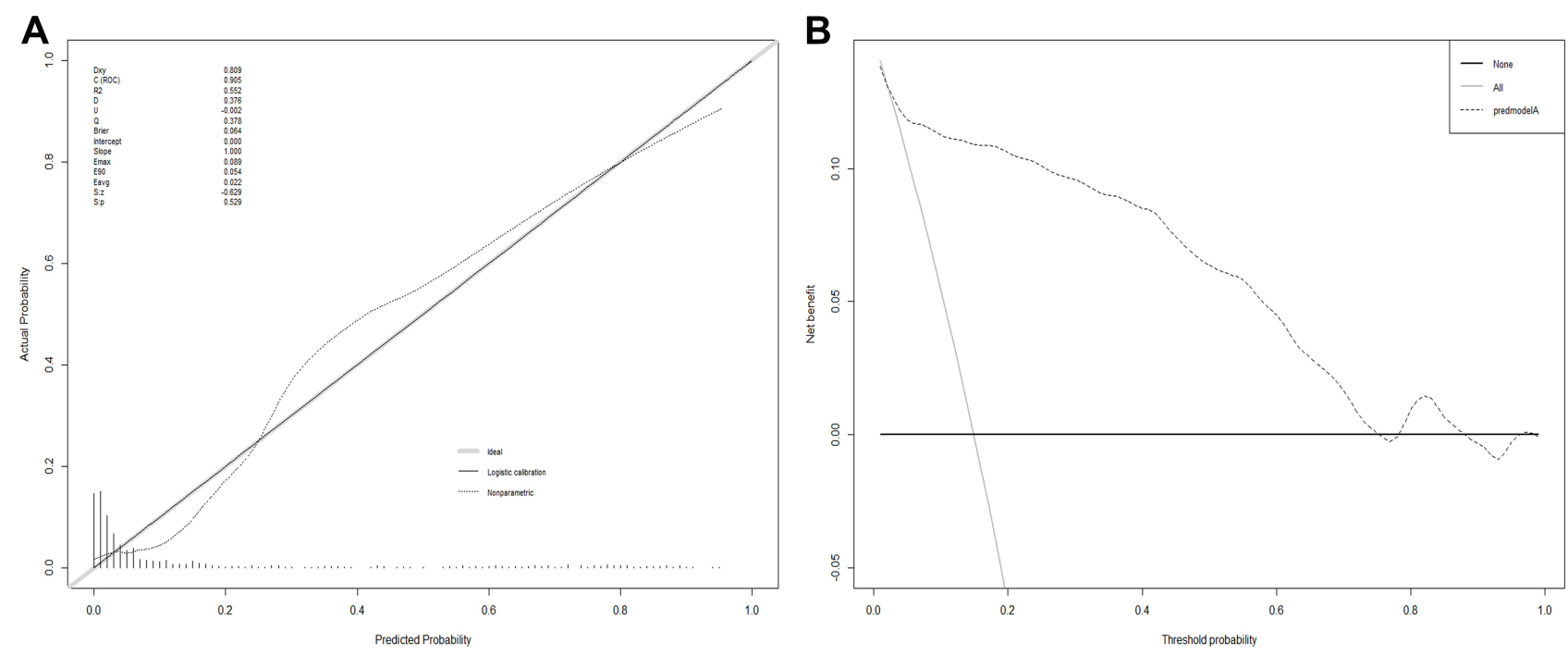

Figure 3 Calibration and decision plot of the nomogram for the probability of postoperative pneumonia. (A) Calibration plot. (B) Decision plot.

Some previous studies had shown that complications after orthopedic surgery were usually associated with higher BMI. ${ }^{24,25}$ However, malnutrition, low BMI and serum albumin had been maintained as risk factors for postoperative pneumonia. ${ }^{20,26,27}$ The reason might be that elderly patients often had difficulty swallowing, which could lead to low body mass index, malnutrition and aspiration pneumonia. And malnourished patients had weak immune defenses and were susceptible to infections. We found that patients with high CRP levels before surgery were prone to postoperative pneumonia. Similarly, Takao et al reported that preoperative high C-reactive protein levels were a risk factor for acute exacerbation of interstitial lung disease after non-pulmonary surgery. ${ }^{28}$ The reason for this phenomenon perhaps was that high CRP induced macrophages to secreted various inflammatory cytokines, such as tumor necrosis factor alpha and interleukin (IL) -1 .

Many studies and guidelines recommended that older patients with hip fractures underwent surgery as soon as possible because early surgery could reduce postoperative complications and mortality. ${ }^{29-32}$ This was consistent with our results. Patients who were delayed in receiving surgical treatment had an increased risk of postoperative pneumonia. Advocating early surgery was beneficial for early restoration of motor function and reduction of complications associated with bed rest. In addition, we found that patients with dependent functional status had an increased probability of developing postoperative pneumonia. Such patients were often elderly and long-term bedridden, which was probably the main reason.

In this study, elderly patients who developed pneumonia after hip fracture had a longer hospital stay, a higher frequency of ICU admission and a higher mortality rate during hospitalization than patients without pneumonia. Taking preventive measures for patients with a high risk of postoperative pneumonia could improve the prognosis process and reduce hospitalization costs. Interventions included maintaining an upright posture while eating, encouraging coughing and deep breathing exercises, raising the head of the bed to at least 30 degrees, encouraging out of bed walking through good pain control, and performing oral hygiene with chlorhexidine twice each day. ${ }^{33}$

There were several limitations in our study. First, this was a retrospective study, and selection bias was unavoidable. Second, we did not collect information on preoperative and intraoperative drug use, perioperative antibiotic use and care programs. Third, the data in this article came from a single center and the amount of data was limited.

\section{Conclusion}

In this study, we found that BMI, CRP, serum albumin, functional status and time to surgery were the independent risk factors for postoperative pneumonia. Our nomogram model can be used to predict the risk of pneumonia in elderly hip fractures after surgery, and provide clinicians with guidance for better perioperative intervention to improve prognosis and reduce mortality. 


\section{Data Sharing Statement}

Patient data comes from our hospital's medical record follow-up database, transparent and available.

\section{Ethical Statement}

The authors are accountable for all aspects of the work in ensuring that questions related to the accuracy or integrity of any part of the work are appropriately investigated and resolved. This study followed the guidelines of the "Declaration of Helsinki" and was approved by the hospital's ethics committee (Research Ethics Committee of the Second Affiliated Hospital of Wenzhou Medical University, L-2020-22). The data were analyzed anonymously and personal identifiers were completely removed; therefore, the requirement for informed consent was waived.

\section{Code Availability}

Statistical analyses were carried out using $\mathrm{R}$ version 3.6.1 for Windows (R Foundation for Statistical Computing, Vienna, Austria) and EmpowerStats (http://www.empower stats.com, X\&Y Solutions, Inc., Boston, MA).

\section{Acknowledgments}

This research was supported by Wenzhou Basic Scientific Research project funding (No. Y20180321), Zhejiang Natural Science Fund project funding (No. LY19H060003). Guangheng Xiang and Xiaoyu Dong are co-first authors for this study.

\section{Author Contributions}

Guangheng Xiang and Yi-Min Weng contributed to the conception and design. Yi-Min Weng, Yongzeng Feng and Jian Xiao contributed to the administrative support. Guangheng Xiang, Tao Xu, Zili He, Chen-Rong Ke and Xiaoyu Dong contributed to the provision of study materials or patients. Guang-Heng Xiang, Yongzeng Feng and Jian Xiao contributed to the collection and assembly of data. Guangheng Xiang, Chen-Rong Ke and Xiaoyu Dong contributed to the data analysis and interpretation. All authors contributed to data analysis, drafting or revising the article, have agreed on the journal to which the article will be submitted, gave final approval of the version to be published, and agree to be accountable for all aspects of the work.

\section{Funding}

This research was supported by Wenzhou Basic Scientific Research project funding (No. Y20180321), Zhejiang Natural Science Fund project funding (No. LY19H060003).

\section{Disclosure}

Guangheng Xiang, Xiaoyu Dong, Tao Xu, Yongzeng Feng, Zili He, Chenrong Ke, Jian Xiao and Yi-Min Weng have no conflicts of interest to disclose in relation to this article. The authors declare no competing financial interests.

\section{References}

1. Tajeu GS, Delzell E, Smith W, et al. Death, debility, and destitution following hip fracture. J Gerontol a Biol Sci Med Sci. 2014;69 (3):346-353. doi:10.1093/gerona/glt105

2. Ha Y-C, Kim T-Y, Lee A, et al. Current trends and future projections of hip fracture in South Korea using nationwide claims data. Osteoporos Int. 2016;27:2603-2609. doi:10.1007/s00198-0163576-9

3. Johansen A, Tsang C, Boulton C, et al. Understanding mortality rates after hip fracture repair using ASA physical status in the National Hip Fracture Database. Anaesthesia. 2007;72(8):961-966. doi:10.1111/anae. 13908

4. Sheehan KJ, Sobolev B, Chudyk A, et al. Patient and system factors of mortality after hip fracture: a scoping review. BMC Musculoskelet Disord. 2016;17(1):166. doi:10.1186/s12891-016-1018-7

5. Chughtai M, Gwam CU, Mohamed N, et al. The epidemiology and risk factors for postoperative pneumonia. J Clin Med Res. 2017;9 (6):466-475. doi:10.14740/jocmr3002w

6. Khan MA, Hossain FS, Ahmed I, et al. Predictors of early mortality after hip fracture surgery. Int Orthop. 2013;37(11):2119-2124. doi:10.1007/s00264-013-2068-1

7. Shigemoto K, Sawaguchi T, Goshima K, et al. The effect of a multidisciplinary approach on geriatric hip fractures in Japan. J Orthop Sci. 2019;24(2):280-285. doi:10.1016/j.jos.2018.09.012

8. Higashikawa T, Shigemoto K, Goshima K, et al. Urinary retention as a postoperative complication associated with functional decline in elderly female patients with femoral neck and trochanteric fractures: a retrospective study of a patient cohort. Medicine. 2019;98(24): e16023. doi:10.1097/MD.0000000000016023

9. Lo IL, Siu CW, Tse HF, et al. Pre-operative pulmonary assessment for patients with hip fracture. Osteoporos Int. 2010;21(Suppl 4): S579-S586. doi:10.1007/s00198-010-1427-7

10. Folbert EC, Hegeman JH, Gierveld R, et al. Complications during hospitalization and risk factors in elderly patients with hip fracture following integrated orthogeriatric treatment. Arch Orthop Trauma Surg. 2017;137(4):507-515. doi:10.1007/s00402-0172646-6

11. Flikweert ER, Wendt KW, Diercks RL, et al. Complications after hip fracture surgery: are they preventable? Eur J Trauma Emerg Surg. 2018;44(4):573-580. doi:10.1007/s00068-017-0826-2

12. Chang SC, Lai JI, Lu MC, et al. Reduction in the incidence of pneumonia in elderly patients after hip fracture surgery: an inpatient pulmonary rehabilitation program. Medicine. 2018;97(33):e11845. doi:10.1097/MD.0000000000011845

13. Lv H, Yin P, Long A, et al. Clinical characteristics and risk factors of postoperative pneumonia after hip fracture surgery: a prospective cohort study. Osteoporos Int. 2016;27(10):3001-3009. doi:10.1007/ s00198-016-3624-5 
14. Byun S-E, Shon H-C, Kim JW, et al. Risk factors and prognostic implications of aspiration pneumonia in older hip fracture patients: a multicenter retrospective analysis. Geriatr Gerontol Int. 2019;19 (2):119-123.

15. Higashikawa T, Shigemoto K, Goshima K, et al. Risk factors for the development of aspiration pneumonia in elderly patients with femoral neck and trochanteric fractures: a retrospective study of a patient cohort Medicine. 2020;99(7):e19108. doi:10.1097/MD.0000000000019108

16. The Japanese Orthopaedic Association. Japanese Society for Fracture Repair. Practice Guideline for Treatment of Femoral Neck and Trochanteric Fractures. Tokyo: NANKODO; 2005.

17. Roche JJ, Wenn RT, Sahota O, et al. Effect of comorbidities and postoperative complications on mortality after hip fracture in elderly people: prospective observational cohort study. BMJ. 2005;331 (7529):1374. doi:10.1136/bmj.38643.663843.55

18. Skull SA, Andrews RM, Byrnes GB, et al. ICD-10 codes are a valid tool for identification of pneumonia in hospitalized patients aged $>$ or = 65 years. Epidemiol Infect. 2008;136(2):232-240. doi:10.1017/ S0950268807008564

19. Jang S-Y, Cha Y, Yoo J-I, et al. Effect of pneumonia on all-cause mortality after elderly hip fracture: a Korean nationwide cohort study. J Korean Med Sci. 2020;35(2):e9. doi:10.3346/jkms.2020.35.e9

20. Langmore SE. Risk factors for aspiration pneumonia. Nutr Clin Pract. 1999;14(4):1-46. doi:10.1177/0884533699014005S09

21. Smetana GW. Preoperative pulmonary evaluation: identifying and reducing risks for pulmonary complications. Cleve Clin $\mathrm{J} \mathrm{Med}$. 2006;73(Suppl 1):S36-S41. doi:10.3949/ccjm.73.Suppl_1.S36

22. Pugely AJ, Martin CT, Gao Y, et al. A risk calculator for short-term morbidity and mortality after hip fracture surgery. J Orthop Trauma. 2014;28(6):3-69. doi:10.1097/BOT.0b013e3182a22744

23. Sheehan KJ, Sobolev B, Guy P, et al. Feasibility of administrative data for studying complications after hip fracture surgery. BMJ Open. 2017;7(4):e015368. doi:10.1136/bmjopen-2016-015368

24. Haynes J, Nam D, Barrack RL. Obesity in total hip arthroplasty: does it make a difference? Bone Joint J. 2017;99-b(1 Supple A):31e6. doi:10.1302/0301-620X.99B1.BJJ-2016-0346.R1
25. Jahng KH, Bas MA, Rodriguez JA, et al. Risk factors for wound complications after direct anterior approach hip arthroplasty. $J$ Arthroplasty. 2016;31(11):2583e7. doi:10.1016/j.arth.2016.04.030

26. Chouinard J, Lavigne E, Villeneuve C. Weight loss, dysphagia, and outcome in advanced dementia. Dysphagia. 1998;13(3):151-155. doi:10.1007/PL00009565

27. Metani H, Tsubahara A, Hiraoka T, et al. Risk factors for patients who develop pneumonia either before or after hip fracture surgery. Japan J Compr Rehabil Sci. 2015;6:43-49.

28. Takao S, Masuda T, Yamaguchi K, et al. High preoperative C-reactive protein level is a risk factor for acute exacerbation of interstitial lung disease after non-pulmonary surgery. Medicine. 2019;98(5):e14296. doi:10.1097/MD.0000000000014296

29. Simunovic N, Devereaux PJ, Sprague S, et al. Effect of early surgery after hip fracture on mortality and complications: systematic review and meta-analysis. CMAJ. 2010;182(15):1609-1616. doi:10.1503/ cmaj.092220

30. Tian M, Gong X, Rath S, et al. Management of hip fractures in older people in Beijing: a retrospective audit and comparison with evidence-based guidelines and practice in the UK. Osteoporos Int. 2016;27(2):677-681. doi:10.1007/s00198-015-3261-4

31. Paul P, Issac RT. Delay in time from fracture to surgery: a potential risk factor for in-hospital mortality in elderly patients with hip fractures. J Orthop. 2018;15(2):375-378. doi:10.1016/j.jor.2018.03.001

32. He W, You YY, Sun K, et al. Admission delay is associated with worse surgical outcomes for elderly hip fracture patients: a retrospective observational study. World J Emerg Med. 2020;11(1):27-32. doi:10.5847/wjem.j.1920-8642.2020.01.004

33. Kazaure HS, Martin M, Yoon JK, et al. Long-term results of a postoperative pneumonia prevention program for the inpatient surgical ward. JAMA Surg. 2014;149(9):914e8. doi:10.1001/ jamasurg.2014.1216
Risk Management and Healthcare Policy

\section{Publish your work in this journal}

Risk Management and Healthcare Policy is an international, peerreviewed, open access journal focusing on all aspects of public health, policy, and preventative measures to promote good health and improve morbidity and mortality in the population. The journal welcomes submitted papers covering original research, basic science, clinical \& epidemiological studies, reviews and evaluations, guidelines, expert opinion and commentary, case reports and extended reports. The manuscript management system is completely online and includes a very quick and fair peer-review system, which is all easy to use. Visit http://www.dovepress.com/testimonials.php to read real quotes from published authors. 